\title{
Bayesian data analysis of the dynamics of rolling leukocytes
}

\author{
M. L. Moskopp ${ }^{a}$, R. Preuss ${ }^{b}$, A. Deussen ${ }^{a}$, \\ T. Chavakis ${ }^{c, a}$, and P. Dieterich ${ }^{a}$ \\ ${ }^{a}$ Institut für Physiologie, \\ Medizinische Fakultät Carl Gustav Carus der TU Dresden, \\ 01307 Dresden, Germany, \\ ${ }^{b}$ Max Planck Institute for Plasma Physics, \\ EURATOM Association, \\ Boltzmannstr. 2, 85748 Garching, Germany \\ ${ }^{c}$ Medizinische Klinik und Poliklinik III, \\ Uniklinikum Dresden,Fetscherstr. 74, \\ 01307 Dresden, Germany
}

January 24, 2014

\begin{abstract}
The coordinated recruitment of leukocytes to sites of infection and inflammation is a central process of the immune system and proceeds in several steps. Here we focus on the dynamics of rolling leukocytes obtained from in vitro experiments. Trajectories of rolling leukocytes in small flow chambers are acquired with phase contrast microscopy under different levels of fluid shear stress and a variation of protein coatings of the (adhesive) surfaces. Bayesian data analysis of a random walk model including drift is applied to individual trajectories of leukocytes. The analysis allows the estimation of drift velocities and diffusion coefficients within an uncertainty of about $10 \%$ and shows a certain homogeneity of the cell groups. Drift velocities of cells saturate in spite of increasing fluid flow. In addition, the analysis reveals some correlated fluctuations of cells' translocations requiring a refinement of the stochastic model.
\end{abstract}




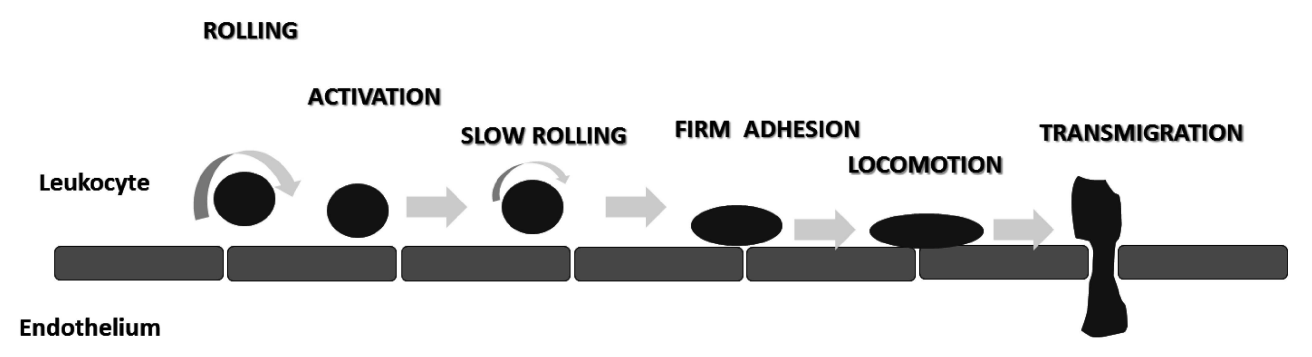

Figure 1: The different steps of the leukocyte adhesion cascade.

\section{Introduction}

The recruitment of leukocytes moving within the blood stream to sites of infection and inflammation is a central process of the immune response [1,2]. The exact adjustment of recruitment of leukocytes is of major importance in physiology and patho-physiology. Thereby this biological system is controlled by a huge number of factors such as biochemical signals, interactions between leukocytes and cells of the blood vessel wall (endothelial cells), or passive influences and stimuli generated by fluid shear stress.

The so-called leukocyte adhesion cascade [3] proceeds in several steps (see Fig. 1): After margination, which means leaving the main blood stream towards the vessel wall into a zone of exponentially down-slowing stream, leukocytes get in touch with wall covering endothelial cells. Dependent on proteins expressed by the endothelium leukocytes start fast and consecutively slow rolling on the endothelium. Whereas fast rolling depends entirely on the interaction between selectins and their carbohydrate ligands, slow rolling is mediated by both selectin- and integrin-dependent interactions [3]. In addition slow rolling is characterized by a decreased mean speed between 5 and $10 \mu \mathrm{m} / \mathrm{sec}$ [4], that allows a simple dynamical differentiation between these two types of rolling. After firm adhesion and spreading on the endothelium leukocytes may start slow crawling like locomotion searching for appropriate sites to perform transmigration into the surrounding tissue. When the leukocyte has left the blood vessel its movement also depends on the 3-dimensional matrix formed by extracellular matrix proteins.

For the following thoughts it is important to know that rolling is only possible in the presence of proteins called selectins, which are expressed and presented by the endothelium. Especially the presence of P-Selectin which interacts with leukocytes' P-Selectin Glycoprotein Ligand 1 (PSGL-1) enables a process called fast rolling.

The dynamics of the bloodstream depend on many factors like the blood 
pressure, the elasticity of the vessel-walls, and the area within the bloodvessel system. It is shown that the adhesion cascade takes place in postcapillary venules, where the shear rate is in a range of $\sim 0.5-5 \mathrm{dyn} / \mathrm{cm}^{2}$.

Here, we focus on the dynamics of fast rolling leukocytes under in-vitro conditions. The dynamics of fast rolling leukocytes is assessed with a simple random walk model including a drift term. Parameter estimation is performed with Bayesian analysis for each trajectory including the covariance matrix of the stochastic process.

\section{Material and methods}

Inside a sterile and closed tube system the monocytic THP1 cells were pumped into slides with P-Selectin coated surfaces. The experimental setup of the whole system is shown in Fig. 2.

\section{Cell culture}

We used the acute monocytic cell line THP-1 as described by Tsuchiya [5]. It was cultured in RPMI 1640 medium supplemented with $10 \%$ fetal bovine serum and $1 \%$ antibiotics containing penicillin and streptomycin at $37^{\circ} \mathrm{C}$ with $5 \% \mathrm{CO}_{2}$. Cells were cultured in tissue culture flask at $1-5 * 10^{5}$ cells $/ \mathrm{mL}$. Two hours before the experiment many flasks were pooled together to reach a concentration of $1 * 10^{6}$ cells $/ \mathrm{mL}$.

\subsubsection{Slides}

The flow chamber slides ( $\mu$-Slide I 0.4 Luer IBIDI-slides, IBIDI - Integrated BioDiagnostics, Martinsried, Germany) have a height of $400 \mu \mathrm{m}$ containing a volume of $100 \mu \mathrm{l}$ inside the channel. A hydrophobic slide was coated with a $100 \mu \mathrm{l}$ solution containing $10 \mu \mathrm{g} / \mathrm{mL}$ P-Selectin, $10 \mu \mathrm{g} / \mathrm{mL}$ ICAM-1 and 1 $\mu \mathrm{g} / \mathrm{mL} \mathrm{MCP}-1$ at $4^{\circ} \mathrm{C}$ over night.

\subsubsection{Experimental setup}

All non-biological materials were left in the incubator around the microscope (see Fig. 2) for 12 hours before starting the experiments to avoid any influence due to variations in temperature on the biological system. The microscope is a Axiovert100M and was operated by the Axiovert-Software to perform phase contrast microscopy. All records were done at a rate of 14 images/secondes $(\Delta t=1 / 14 s)$ with a $10 \mathrm{x}$ objective lens. The records have a length of up to 140 images corresponding to a time interval of 10 seconds. We 


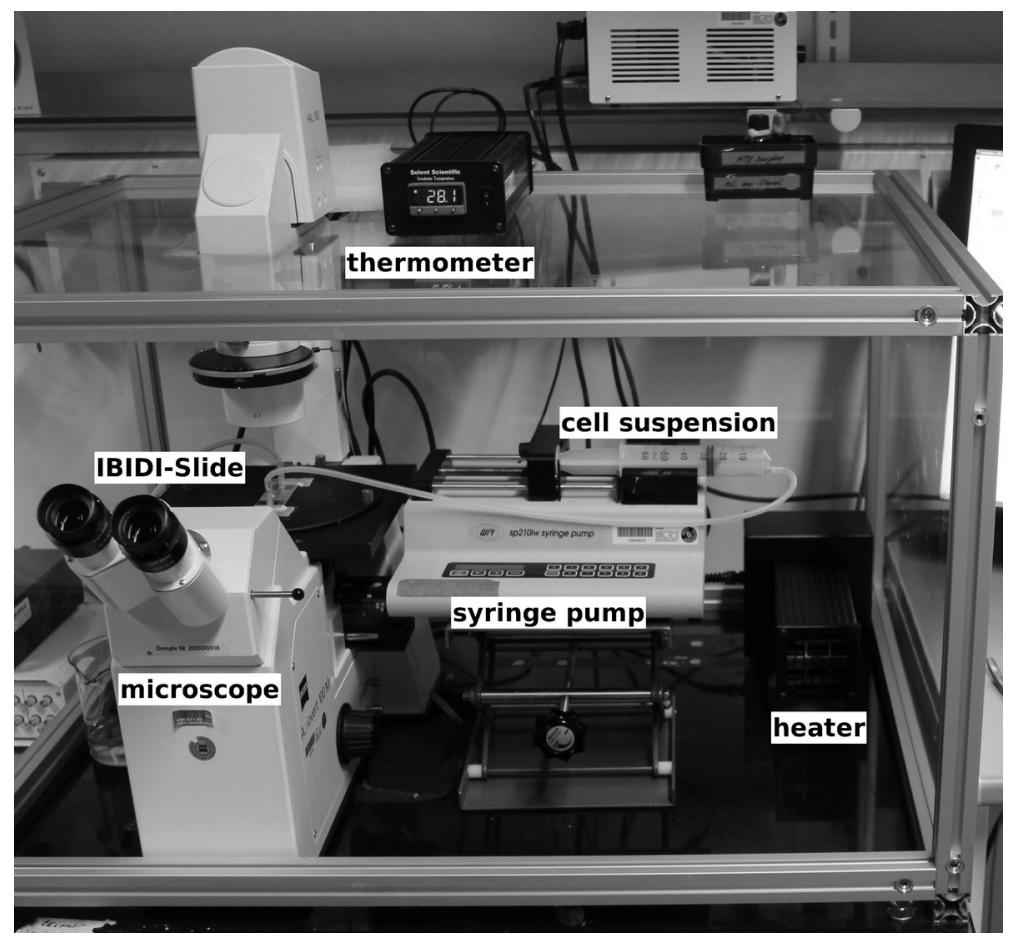

Figure 2: Monocyte-like THP1 cells were put inside a tube system [cell suspension] and pumped into small flow chamber [IBIDI-Slide] where surfaces were coated with proteins. The system is transparent and cells were observed with a phase contrast microscope. Time-lapse sequences of these experiments were used to assess the dynamics of rolling leukocytes under different levels of fluid shear stress.

used a syringe pump type WPI sp210iw, which was manually programmed. The links between applied flow and resulting shear rates within the channel were calculated with the given formula from IBIDI. Within this experimental setting shear rates between 0.1 and $10 \mathrm{dyn} / \mathrm{cm}^{2}$ were used to simulate the conditions of postcapillary venules. In every case the pump was stopped for 1 minute between two records to ensure that enough THP-1 cells sank to the coated bottom of the slide.

\subsubsection{Image segmentation}

The segmentation was done with own image-processing programs applying a combination of edge and size detection which is illustrated in Fig. 3. The obtained contours were used to maintain the paths of individual cells as a function of time. 


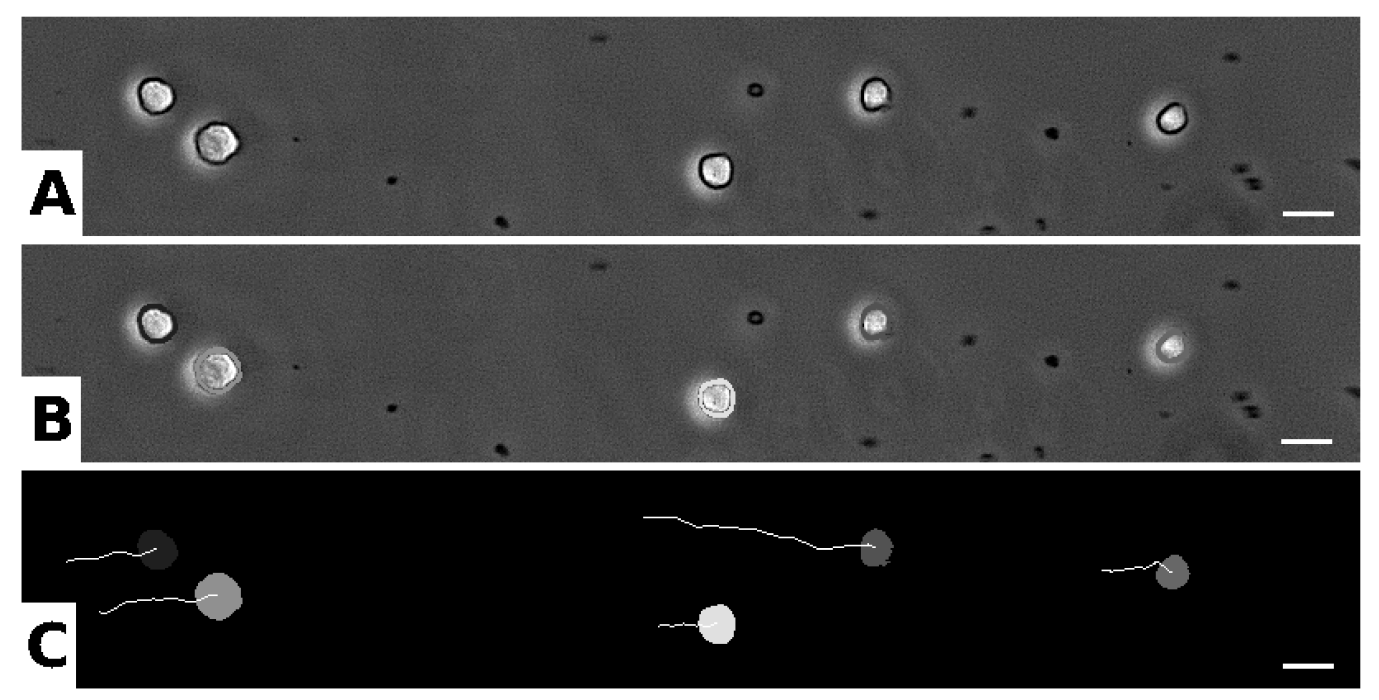

Figure 3: Paths of segmented THP-1 cells on P-Selectin coated surface under shear stress $1 \mathrm{dyn} / \mathrm{cm}^{2}$ (from left to right). Cells were observed for 2 seconds $(\Delta t=71.4 \mathrm{~ms})$ during fast rolling. The white length bar indicates $20 \mu \mathrm{m}$. A - native record. B - segmented edges. C - cell paths within 2 seconds.

\section{Bayesian data analysis of rolling leukocytes}

\subsection{Technique}

Inspection of raw trajectories showed that the dynamics of rolling leukocytes are mainly dominated by a constant drift and additional small fluctuations. Therefore, we first assumed a random walk model (RW) with a constant drift term $v_{d}$ (generated by the external flow) and a diffusion term. The position of a cell $x_{i}$ at time $t_{i}=i \Delta t$ can be written as

$$
x_{i+1}=x_{i}+\sqrt{2 D \Delta t} \xi_{i+1}+v_{d} \Delta t
$$

with uncorrelated random Gaussian variables $\xi_{i} \sim N(0,1) . \Delta t$ denotes the sampling time interval. Expanding the discrete form of Eq. 1 for all positions $i=0 \ldots L$ leads to a compact matrix notation of the process:

$$
\left(\begin{array}{c}
x_{1}-x_{0} \\
x_{2}-x_{0} \\
x_{3}-x_{0} \\
\vdots \\
x_{L}-x_{0}
\end{array}\right)=\sqrt{2 D \Delta t}\left(\begin{array}{ccccc}
1 & & & & \\
1 & 1 & & 0 & \\
1 & 1 & 1 & & \\
\vdots & \vdots & \vdots & \ddots & \\
1 & 1 & 1 & \ldots & 1
\end{array}\right) \cdot\left(\begin{array}{c}
\xi_{1} \\
\xi_{2} \\
\xi_{3} \\
\vdots \\
\xi_{L}
\end{array}\right)+v_{d} \Delta t\left(\begin{array}{c}
1 \\
2 \\
3 \\
\vdots \\
L
\end{array}\right)
$$

This representation clearly shows that positions $x_{i}$ are correlated along the path. As mean and variance of $x_{i}$ can be calculated by integration over all 
$\xi_{i}$ (see the contributions of Dieterich and Preuss to these proceedings $[6,7]$ ) the likelihood for data $\left\{x_{i}\right\}$ of the model in Eq. 2 is given by

$$
P\left(x_{i} \mid v_{d}, D, I\right)=\frac{1}{\sqrt{(2 \pi)^{L} \operatorname{det} \mathcal{C}}} \exp \left\{-\frac{1}{2} \sum_{i, j}^{L}\left(x_{i}-v_{d} t_{i}\right)\left[\mathcal{C}^{-1}\right]_{i, j}\left(x_{j}-v_{d} t_{j}\right)\right\}
$$

The covariance matrix $\mathcal{C}$ is identical to the aging correlation function of positions, which can be calculated as $\left\langle\left[x\left(t_{i}\right)-v_{d} t_{i}\right]\left[x\left(t_{j}\right)-v_{d} t_{j}\right]\right\rangle=2 D \Delta t \min (i, j)$ for the random walk process. It is directly related to the matrix in Eq. 2 multiplying it with its transpose.

\section{$3.2 \quad$ Results}

The likelihood of Eq. 3 together with the theoretical covariance for the random walk process is used to obtain the estimation of parameters and their uncertainties for each trajectory of leukocytes under different experimental conditions.

The estimation of drift velocities of cells as a function of increasing fluid flow (shear stress) saturates between 35 and $40 \mu \mathrm{m} / \mathrm{s}$ as shown in Fig. 4 . The analysis allows the estimation of drift velocities and diffusion coefficients within an uncertainty of about $10 \%$ as shown in Fig. 5. In addition, the analysis shows a certain homogeneity of the cell groups.

\section{Discussion}

The Bayesian data analysis provides a good estimation of parameters of the assumed RW model. However, the analysis of trajectories in Fig. 6 reveals some additional correlated fluctuations not included in the RW model. This observation is supported by the velocity autocorrelation function decaying within 2 to 3 time intervals $\Delta t$. These intermittent fluctuations appeared in almost all cells in an asynchronous manner and could be imagined as stick-slip processes.

The fact that we were able to identify two different groups of cells according to their estimated diffusion constants $\langle D\rangle$ raises even more questions about the observed process (see Fig. 7). At this time we do not know the reasons for the separation into these two groups. As we are using a monoclonal cell line influences due to genetic diversity should be small and not causative for this effect. Possibly the individual time span since the first contact of each cell to the presented protein plays an important role.

In summary, the presented formalism allowed to study the dynamics of rolling leukocytes with a random walk model including a linear drift at the 


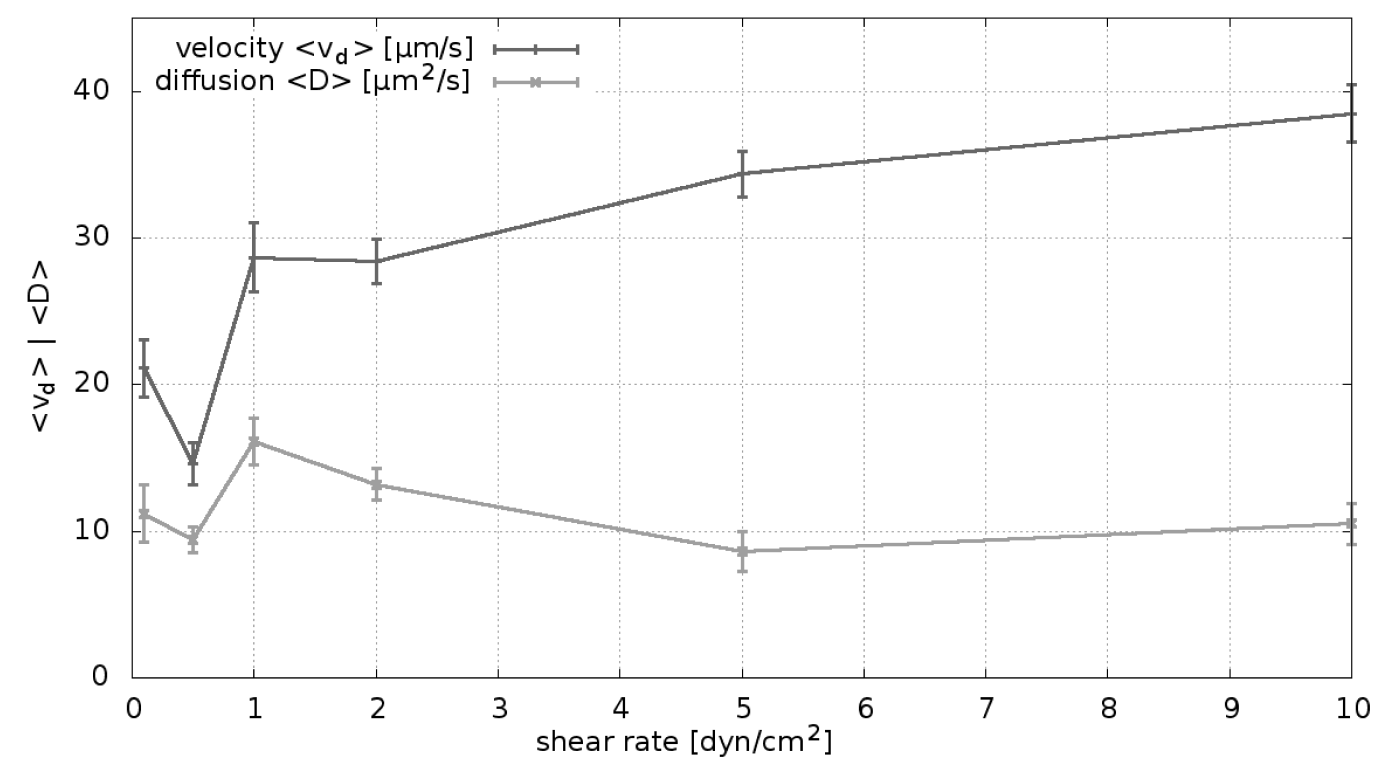

Figure 4: The figure shows the estimated cell velocities and diffusion coefficients for different fluid shear rates. It is remarkable that velocities saturate between $35 \mu \mathrm{m} / \mathrm{s}$ and $40 \mu \mathrm{m} / \mathrm{s}$ although flow velocity is increased linearly with shear rate. 


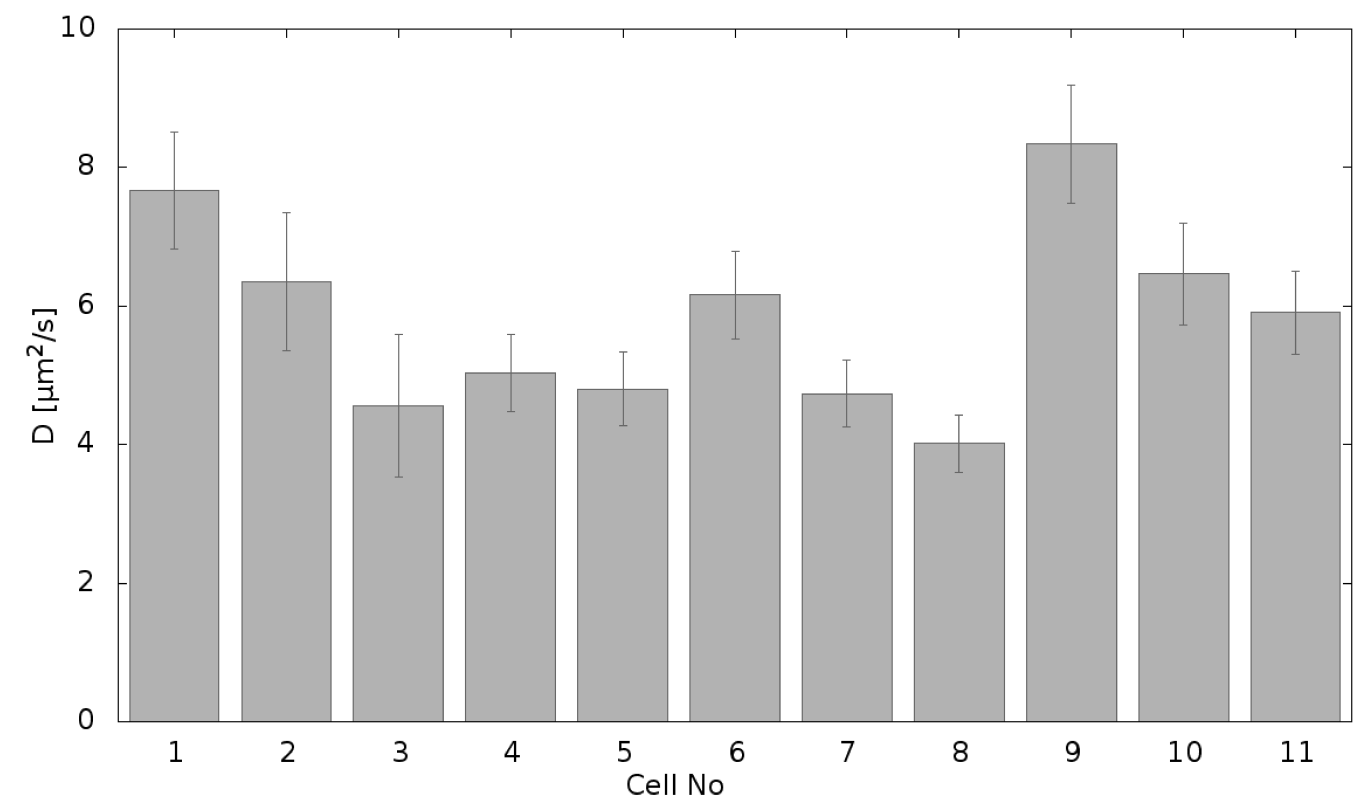

Figure 5: Diffusion constants $D$ are estimated for eleven different cells at shear stress $2 \mathrm{dyn} / \mathrm{cm}^{2}$. The analysis shows that the coefficients can be estimated with errors of about $10 \%$. The group of these eleven cells shows diffusion coefficients below $10 \mu \mathrm{m}^{2} / \mathrm{s}$ whereas an other subgroup has diffusion coefficient above this value. This enables a division of the cells into two groups (see Fig. 7). 


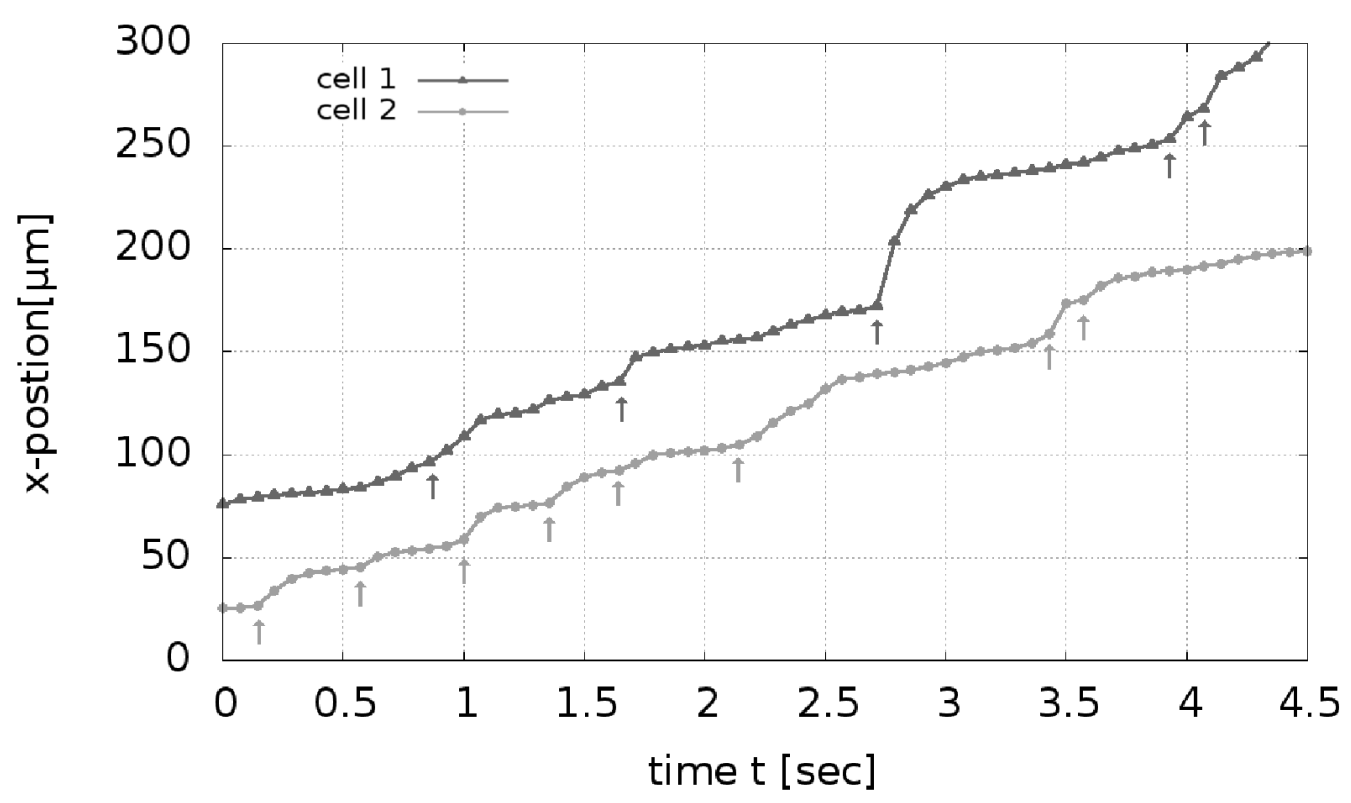

Figure 6: Cell trajectories show small fluctuations around the mean constant drift behavior. This effect appeared in almost all cells in an asynchronous manner.

level of a single cell. The formalism included the so-called aging correlations in a consistent way and can be used to study more complicated stochastic models with additional features missing in the random walk model. In addition, we will apply this kind of Bayesian data analysis to further experiments concentrating on other parts of the leukocytes adhesion cascade as shown in Fig. 1.

\section{References}

[1] C. Auffray, D. Fogg, M. Garfa, G. Elain, O. Join-Lambert, S. Kayal, S. Sarnacki, A. Cumano, G. Lauvau, F. Geissmann, Science 317(5838):666670 (2007).

[2] T. Chavakis, M. Hussain, S. Kanse, G. Peters, R. Bretzel, J.I. Flock, M. Herrmann, K.T. Preissner, Nature Medicine 8:687-693 (2002).

[3] K. Ley, C. Laudanna, M.I. Cybulsky, S. Nourshargh, Nat Rev Immunol. 7(9):678-689 (2007).

[4] U. Jung, K. Ley, J Immunol. 162(11):6755-6762 (1999). 


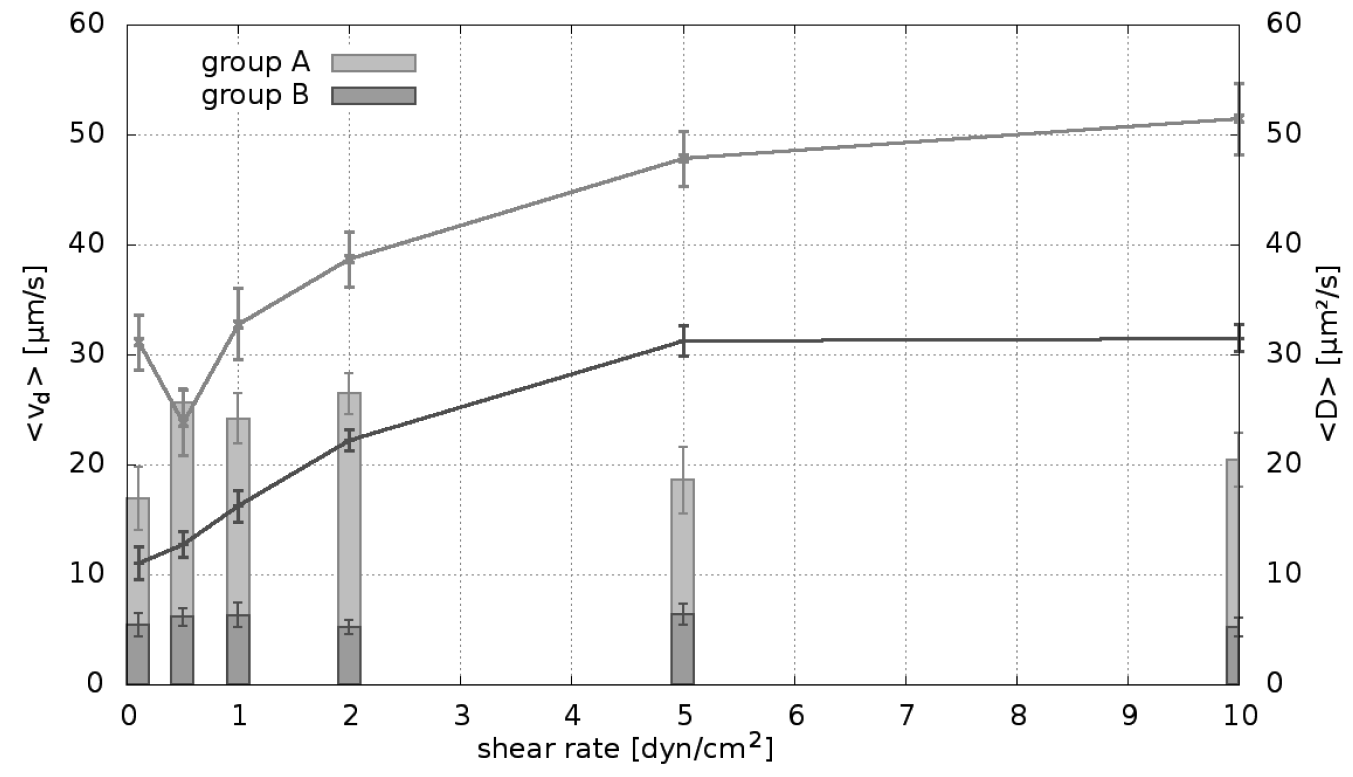

Figure 7: Estimated velocities and diffusion constants for different fluid shear rates. Here cells are divided into two groups: Group A with estimated diffusion coefficients $D<10 \mu \mathrm{m}^{2} / \mathrm{s}$ and group B with estimated diffusion coefficients $D>10 \mu \mathrm{m}^{2} / \mathrm{s}$. Both velocity curves are saturating (group A between $50 \mu \mathrm{m} / \mathrm{s}$ and $55 \mu \mathrm{m} / \mathrm{s}$; group B at $\sim 32 \mu \mathrm{m} / \mathrm{s}$ ) although flow velocity is increased linearly with shear rate. 
[5] S. Tsuchiya, M. Yamabe, Y. Yamaguchi, Y. Kobayashi, T. Konno, K. Tada, Int J Cancer. 26(2):171-176 (1980).

[6] P. Dieterich, R. Preuss (2012): Bayesian parameter estimation for stochastic models of biological cell migration. In Proceedings of the 32nd International Workshop on Bayesian Inference and Maximum Entropy Methods in Science and Engineering, Garching.

[7] R. Preuss, P. Dieterich (2012): Employment of the covariance matrix in parameter estimation for stochastic processes in cell biology. In Proceedings of the 32nd International Workshop on Bayesian Inference and Maximum Entropy Methods in Science and Engineering, Garching. 